\title{
Mitochondrial DNA copy number in peripheral blood leukocytes and the aggressiveness of localized prostate cancer
}

\author{
Huakang Tu${ }^{1}$, Jian $\mathrm{Gu}^{1}$, Qing H. Meng ${ }^{2}$, Jeri Kim ${ }^{3}$, John W. Davis ${ }^{4}$, Yonggang $\mathrm{He}^{5}$, \\ Elizabeth A. Wagar ${ }^{2}$, Timothy C. Thompson ${ }^{3}$, Christopher J. Logothetis ${ }^{3}$, Xifeng $\mathbf{W u}^{1}$ \\ ${ }^{1}$ Department of Epidemiology, The University of Texas MD Anderson Cancer Center, Houston, Texas, USA \\ ${ }^{2}$ Department of Laboratory Medicine, The University of Texas MD Anderson Cancer Center, Houston, Texas, USA \\ ${ }^{3}$ Department of Genitourinary Medical Oncology, The University of Texas MD Anderson Cancer Center, Houston, Texas, USA \\ ${ }^{4}$ Department of Urology, The University of Texas MD Anderson Cancer Center, Houston, Texas, USA \\ ${ }^{5}$ Department of Surgery, Ruijin Hospital, Shanghai Jiao Tong University School of Medicine, Shanghai, China \\ Correspondence to: \\ Xifeng Wu, e-mail: xwu@mdanderson.org \\ Keywords: mitochondrial DNA copy number, prostate cancer, aggressiveness, recurrence, progression \\ Received: August 19, $2015 \quad$ Accepted: October 16, 2015 \\ Published: October 26, 2015
}

\section{ABSTRACT}

We investigated whether low mitochondrial DNA copy number (mtDNAcn) in peripheral blood leukocytes at diagnosis was associated with an increased risk of the aggressive form of the tumor and disease progression among localized prostate cancer ( $\mathrm{PCa}$ ) patients. We recruited 1,751 non-Hispanic white men with previously untreated PCa from The University of Texas MD Anderson Cancer Center. mtDNAcn was categorized into three groups according to tertiles. We used multivariate logistic regression to estimate the odds ratios (ORs) and 95 percent confidence intervals ( $95 \%$ CIs) for the association of mtDNAcn with the risk of having aggressive PCa at diagnosis. We used Cox proportional hazards model to estimate hazard ratios (HRs) and $95 \%$ CIs for disease progression. We observed an inverse association between aggressiveness of PCa and mtDNAcn $(P<0.001)$. In multivariate analysis, compared to patients in the highest tertile of mtDNAcn, those in the second and lowest tertiles had significantly increased risks of presenting with the high-risk form of PCa, as defined by the D'Amico criteria, with ORs of $1.33(95 \% \mathrm{CI}, 0.89-1.98 ; P=0.17)$ and $1.53(95 \%$ CI, 1.02-2.30; $P=0.04)$, respectively. Furthermore, PCa patients in the lowest and second tertiles combined relative to those in the highest tertile had a 56\% increased risk of disease progression (HR, 1.56; 95\% CI, 0.96-2.54; $P=0.07$ ). In summary, our results suggested that low mtDNAcn in peripheral blood leukocytes was associated with aggressive $\mathrm{PCa}$ at diagnosis and might further predict poor progression-free survival among localized PCa patients.

\section{INTRODUCTION}

Mitochondria are key organelles that perform multiple central cellular functions such as energy production, cell proliferation, and apoptosis $[1,2]$. Besides the nuclei, mitochondria are the only organelles in human cells that possess their own DNA (mtDNA). Each mitochondrion contains $1-15$ mtDNA molecules $[3,4]$, and the number of mitochondria per cell ranges from several hundred to greater than 10,000 mainly depending on the energy demand of the cell as well as environmental factors $[5,6]$. In comparison to nuclear DNA, the mutation rate of mtDNA is about 10 -fold higher due to the lack of introns and protective histones, limited DNA repair mechanisms, and its close proximity to the electron transport chain which generates reactive oxygen species $[3,7-10]$. Alterations in mtDNA including mutations and changes in copy number have been associated with multiple cancers including prostate cancer (PCa) [11-18].

$\mathrm{PCa}$, the most prevalent cancer among men in the US, accounts for $26 \%$ of all cancer cases in men [19]. When diagnosed at an early stage (i.e., localized or regional) the 5 -year survival rate is almost $100 \%$, but when the tumor has metastasized, the rate is only $28.0 \%$ [20]. 
The blood prostate-specific antigen (PSA) test is the most commonly used screening test for PCa. However, the PSA test often captures asymptomatic indolent tumors that have little or no lethal potential, thus causing over-diagnosis and over-treatment $[19,21]$. In order to distinguish between indolent and aggressive $\mathrm{PCa}$, multiple pre-treatment risk stratification systems have been proposed [22]. The most commonly used one is the D'Amico risk criteria, which groups localized PCa patients into three categories: low-risk, intermediate-risk, and high-risk based on total Gleason score, clinical stage and PSA levels at diagnosis [23]. However, these groups are still heterogeneous and do not explain all the variations seen in prognosis, treatment and management [22]; therefore, new markers for disease aggressiveness and progression are needed.

Multiple in vitro cell line studies showed that low mtDNA copy number (mtDNAcn) was associated with aggressive phenotypes of PCa [24-26], suggesting that mtDNAcn could be a potential biomarker for distinguishing indolent from aggressive PCa. However, evidence from human studies is very limited. Three previous human population studies on mtDNAcn and aggressive features of PCa at diagnosis produced conflicting results [26-28]. Regarding disease progression, to the best of our knowledge, no population study has prospectively investigated whether mtDNAcn at diagnosis is associated with disease progression of PCa.

In this study, we investigated whether low mtDNAcn in peripheral blood leukocytes was associated with aggressive features of $\mathrm{PCa}$ in a large cohort of patients with localized PCa. Furthermore, we investigated whether low mtDNAcn could predict disease progression independent of the D'Amico risk stratification system.

\section{RESULTS}

\section{mtDNA copy number in peripheral blood leukocytes by selected characteristics of the study population}

The distribution of selected characteristics of the study participants and mtDNAcn levels by selected characteristics are shown Table 1. The median follow-up time was 44.3 (range: $0.3-156.8$ ) months. The majority of the participants were between 55 and 65 years old, overweight or obese, non-smokers or former smokers, had D'Amico intermediate-risk form of tumor, had a total Gleason score of 7, had T1 stage tumor, and had PSA at diagnosis $<10 \mathrm{ng} / \mathrm{ml}$. Radical prostatectomy $(51.7 \%)$ was the most common treatment. mtDNAcn was significantly lower among patients who were older $(P<0.001)$ or obese $(P=0.04)$. Non-smokers seemed to have lower mtDNAcn but the difference was not statistically significant $(P=0.74)$. mtDNAcn decreased as the aggressiveness (defined by the D'Amico risk groups) increased $(P<0.001)$. In addition, mtDNAcn decreased as total Gleason score increased $(P=0.009)$, as tumor stage increased $(P=0.08)$, and as PSA levels at diagnosis increased $(P=0.21)$. mtDNAcn did not differ significantly among patients receiving different initial primary treatments $(P=0.54)$.

\section{mtDNA copy number and aggressiveness of localized prostate cancer}

The associations of mtDNAcn with the risk of presenting with the intermediate- and high-risk form of PCa are shown in Table 2. In univariate analysis, compared to patients in the highest tertile of mtDNAcn group, the ORs of presenting with high-risk form of $\mathrm{PCa}$ for patients in the second and lowest tertiles were 1.44 (95\% CI, 1.03-2.03; $P=0.04)$ and 1.96 (95\% CI, $1.40-2.75 ; P<0.001)$, respectively; the associations were attenuated but remained statistically significant after adjustment of age, BMI, smoking status and pack-year (second vs. highest tertile: OR, 1.33; 95\% CI, 0.89-1.98; $\mathrm{p}=0.17$; lowest vs. highest tertile: OR, 1.53; 95\% CI, $1.02-2.30 ; P=0.04 ; P$ for trend $=0.047)$. When we conducted stratified analysis by age $(<60 v s . \geq 60$ years $)$, BMI ( $<30$ vs. $\geq 30 \mathrm{~kg} / \mathrm{m}^{2}$ ), and smoking status (neversmokers vs. ever-smokers), the results were similar (Supplementary Table 1). Meanwhile, mtDNAcn was not significantly associated with the intermediate-risk form of PCa. In addition, we used the restricted cubic spline to model the potential non-linear association of mtDNAcn with the high-risk form of PCa. As shown in Figure 1, a lower mtDNAcn was associated with a higher risk of presenting with the high-risk form of $\mathrm{PCa}$, but the effect seems to plateau at mtDNAcn of 0.75 .

We further investigated whether mtDNAcn was associated with Gleason score overall and by PSA levels (Supplementary Table 2). Overall, low mtDNAcn was borderline significantly associated with a Gleason score $\geq 8$ (lowest $v s$. highest tertile: OR, 1.53; 95\% CI, 0.97-2.43; $P=0.07$ ), and the association was only present among patients with PSA $\leq 4 \mathrm{ng} / \mathrm{ml}$ (OR, 4.88; 95\% CI, 1.30-18.39; $P=0.02$ ).

\section{mtDNA copy number and disease progression of localized prostate cancer}

The unadjusted and adjusted associations of mtDNAcn with disease progression among patients who received active treatments are shown in Figure 2 and Table 3 . Since the rate of disease progression is relatively low among patients with localized PCa, we combined the lowest and second tertiles for the analysis of disease progression to increase statistical power. As shown in Figure 2, compared to patients in the highest tertile, patients in the first and second tertiles combined had worse progression-free survival $(P=0.02)$. In crude Cox regression analysis (Table 3 ), compared to $\mathrm{PCa}$ patients in the highest tertile of mtDNAcn group, those in the second 
Table 1: mtDNAcn in peripheral blood leukocytes by selected characteristics of the study patients with localized prostate cancer

\begin{tabular}{|c|c|c|c|}
\hline Characteristics $^{\mathrm{a}}$ & Total $(N=1,751)$ & mtDNAcn $^{b}$ & $P$ value $^{\mathrm{c}}$ \\
\hline \multicolumn{4}{|l|}{ Age at diagnosis, years } \\
\hline$<55$ & $339(19.4)$ & $0.87(0.37)$ & \\
\hline $55-65$ & $836(47.7)$ & $0.81(0.36)$ & \\
\hline$>65$ & $576(32.9)$ & $0.75(0.33)$ & $<0.001$ \\
\hline \multicolumn{4}{|l|}{ BMI at diagnosis, $\mathrm{kg} / \mathrm{m}^{2}$} \\
\hline$<25$ & $256(17.6)$ & $0.79(0.33)$ & \\
\hline 25-29.99 (overweight) & $674(46.3)$ & $0.81(0.36)$ & \\
\hline$\geq 30$ (obese) & $527(36.2)$ & $0.76(0.30)$ & 0.04 \\
\hline \multicolumn{4}{|l|}{ Smoking status at diagnosis } \\
\hline Non-smoker & $819(47.0)$ & $0.80(0.35)$ & \\
\hline Former smoker & $782(44.9)$ & $0.81(0.35)$ & \\
\hline Current smoker & $140(8.0)$ & $0.82(0.38)$ & 0.74 \\
\hline \multicolumn{4}{|l|}{ D'Amico risk group } \\
\hline Low & $589(33.6)$ & $0.83(0.38)$ & \\
\hline Intermediate & $830(47.4)$ & $0.81(0.34)$ & \\
\hline High & $332(19.0)$ & $0.74(0.31)$ & $<0.001$ \\
\hline \multicolumn{4}{|l|}{ Total Gleason score } \\
\hline$\leq 6$ & $647(37.0)$ & $0.83(0.37)$ & \\
\hline 7 & $881(50.3)$ & $0.80(0.34)$ & \\
\hline$\geq 8$ & $222(12.7)$ & $0.74(0.31)$ & 0.009 \\
\hline \multicolumn{4}{|l|}{ Clinical tumor stage } \\
\hline $\mathrm{T} 1$ & $1,095(62.5)$ & $0.82(0.36)$ & \\
\hline $\mathrm{T} 2$ & $569(32.5)$ & $0.79(0.35)$ & \\
\hline $\mathrm{T} 3-\mathrm{T} 4$ & $74(4.2)$ & $0.74(0.25)$ & 0.08 \\
\hline \multicolumn{4}{|l|}{ PSA at diagnosis } \\
\hline$<10 \mathrm{ng} / \mathrm{ml}$ & $1,527(87.4)$ & $0.81(0.36)$ & \\
\hline $10-20 \mathrm{ng} / \mathrm{ml}$ & $154(8.8)$ & $0.77(0.29)$ & \\
\hline$>20 \mathrm{ng} / \mathrm{ml}$ & $67(3.8)$ & $0.75(0.31)$ & 0.21 \\
\hline \multicolumn{4}{|l|}{ Initial primary treatment } \\
\hline Radical prostatectomy & $906(51.7)$ & $0.82(0.36)$ & \\
\hline Radiotherapy & $376(21.5)$ & $0.79(0.34)$ & \\
\hline Surveillance or unknown $^{\mathrm{d}}$ & $427(24.4)$ & $0.80(0.36)$ & \\
\hline Other treatment ${ }^{\mathrm{e}}$ & $42(2.4)$ & $0.80(0.36)$ & 0.55 \\
\hline
\end{tabular}

${ }^{\text {aReported as count (percentage) }}$

${ }^{\mathrm{b}}$ Reported as mean (standard deviation)

'For the differences in mtDNAcn by selected characteristics using ANOVA

dPatients undergoing active surveillance/watchful waiting or whose initial treatment information was unavailable

${ }^{\mathrm{e}}$ Chemotherapy, cryoablation, high-intensity focused ultrasound, or transurethral resection of prostate

Abbreviations: mtDNAcn, mitochondrial DNA copy number; BMI, body mass index; PSA, prostate-specific antigen. 
Table 2: mtDNAcn in peripheral blood leukocytes and aggressiveness of localized prostate cancer at diagnosis

\begin{tabular}{|c|c|c|c|c|c|c|c|}
\hline \multirow{2}{*}{ mtDNAcn } & \multirow{2}{*}{$\begin{array}{c}\text { Low-riskform } \\
\text { of PCa } \\
N(\%)\end{array}$} & \multicolumn{3}{|c|}{ Intermediate-riskform of $\mathrm{PCa}$} & \multicolumn{3}{|c|}{ High-riskform of Pca } \\
\hline & & $N(\%)$ & $\begin{array}{l}\text { Adjusted OR } \\
(95 \% \mathrm{CI})\end{array}$ & $P$ value & $N(\%)$ & $\begin{array}{l}\text { Adjusted OR } \\
\text { (95\% CI) }\end{array}$ & $P$ value \\
\hline $\begin{array}{l}3^{\text {rd }} \text { tertile } \\
\text { (highest) }\end{array}$ & $214(36.3)$ & $285(34.3)$ & Reference & $\mathrm{N} / \mathrm{A}$ & $84(25.3)$ & Reference & N/A \\
\hline $2^{\text {nd }}$ tertile & $201(34.1)$ & $269(32.4)$ & $0.89(0.66-1.19)$ & 0.42 & $114(34.3)$ & $1.33(0.89-1.98)$ & 0.17 \\
\hline $\begin{array}{l}1^{\text {st }} \text { tertile } \\
\text { (lowest) }\end{array}$ & $174(29.5)$ & $276(33.3)$ & $1.05(0.78-1.42)$ & 0.75 & $134(40.4)$ & $1.53(1.02-2.30)$ & 0.04 \\
\hline$P$ for trend & & & 0.77 & & & 0.047 & \\
\hline
\end{tabular}

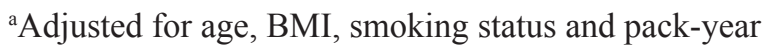

Abbreviations: mtDNAcn, mitochondrial DNA copy number; PCa, prostate cancer; OR, odds ratio; CI, confidence interval

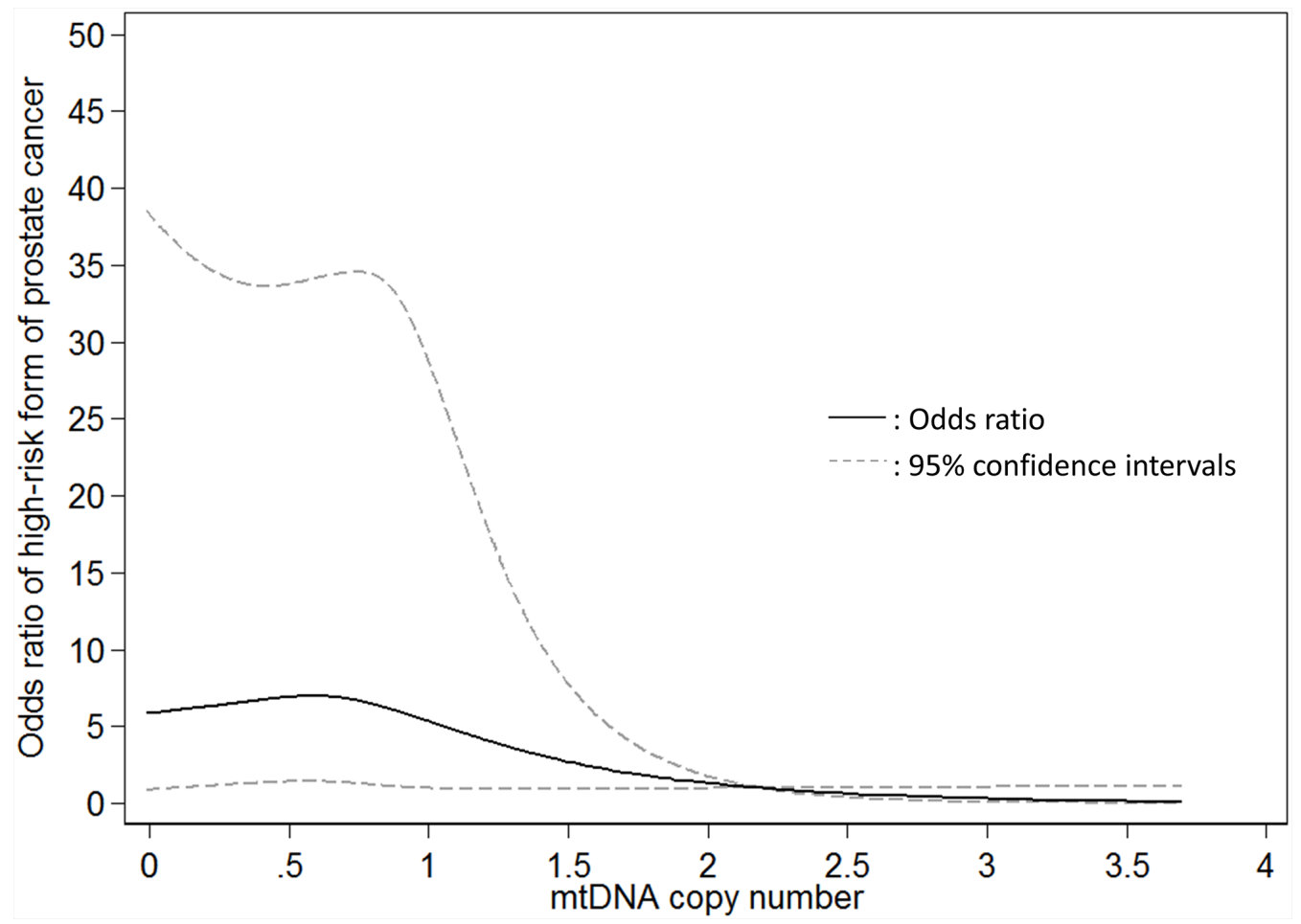

Figure 1: Restricted cubic spline modelling for the association of mitochondrial DNA copy number in peripheral blood leukocytes with high-risk form of localized prostate cancer in comparison to low-risk form of prostate cancer. Four knots were used and the 99th percentile (2.195) was chose as the reference level.

and lowest tertiles combined had an increased risk of disease progression with a HR of 1.59 (95\% CI, 1.09-2.34; $P=0.02)$; after adjustment of age, BMI, smoking status, and pack-year, D'Amico risk groups, and treatments, the multivariate-adjusted $\mathrm{HR}$ was similar and borderline significant (HR, 1.56; 95\% CI, 0.96-2.54; $P=0.07)$. We also conducted stratified analysis by age, BMI, smoking status, and D'Amico risk groups (Supplementary Table 3), and there was no evidence of heterogeneity between different strata.

\section{DISCUSSION}

In this study of 1,751 patients with localized $\mathrm{PCa}$, we found that low mtDNAcn in peripheral blood leukocytes was associated with aggressive features of $\mathrm{PCa}$ at diagnosis including higher D' Amico risk groups and higher total Gleason score; in addition, our results suggested that low mtDNAcn might be associated with an increased risk of disease progression independent of the D' Amico risk stratification groups. 


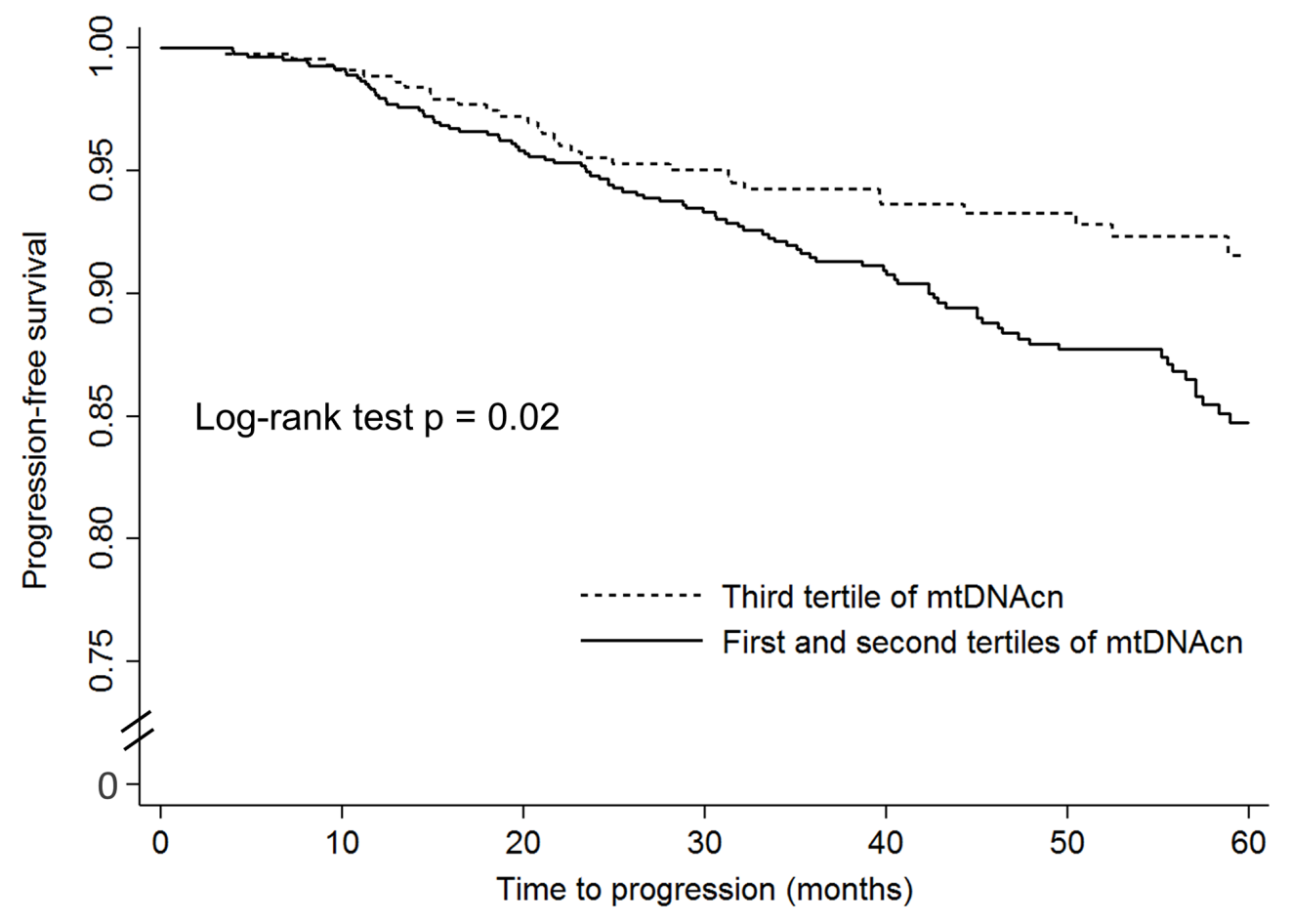

Figure 2: The Kaplan-Meier progression-free survival curves by mitochondrial DNA copy number in peripheral blood leukocytes among localized prostate cancer patients who received active treatments.

Table 3: mtDNAcn in peripheral blood leukocytes and disease progression among localized prostate cancer patients who received active treatments

\begin{tabular}{|l|c|c|c|c|c|c|}
\hline \multicolumn{1}{c}{ mtDNAcn } & $\begin{array}{c}\text { Progression } \\
\boldsymbol{N}(\%)\end{array}$ & $\begin{array}{c}\text { No Progression } \\
\boldsymbol{N}(\%)\end{array}$ & $\begin{array}{c}\text { Crude HR } \\
(95 \% \text { CI) }\end{array}$ & \multicolumn{2}{c}{$\begin{array}{c}\text { P value } \\
\text { Adjusted HR } \\
(95 \% \text { CI) }\end{array}$} \\
\hline $3^{\text {rd }}$ tertile (highest) & $35(8.0)$ & $401(92.0)$ & Reference & N/A & Reference & N/A \\
\hline $2^{\text {nd }}$ and $1^{\text {st }}$ tertile & $104(12.5)$ & $726(87.5)$ & $1.59(1.09-2.34)$ & 0.02 & $1.56(0.96-2.54)$ & 0.07 \\
\hline
\end{tabular}

aAdjusted for age, BMI, smoking status, pack-year, D'Amico risk groups, and initial primary treatment Abbreviations: mtDNAcn, mitochondrial DNA copy number; HR, hazard ratio; CI, confidence interval

Our findings are consistent with previous cell line studies which showed that low mtDNAcn was associated with aggressive phenotype of PCa cells [24-26]. Also, our study is consistent with human studies on other cancers including gastric [29], ovarian [30], renal [31], and breast [32] cancers, which showed that low mtDNAcn in tumor tissue was associated with aggressive features of the tumor. Three previous human studies on mtDNAcn and aggressive features of $\mathrm{PCa}$ reported inconsistent results [26-28]. The first study $(n=14)$ found that low mtDNAcn in PCa tumor tissue was associated with high-grade tumors (Gleason grade 8 and 9) [26]. The second study $(n=38)$ showed mtDNAcn in PCa tumor tissue or peripheral blood leukocytes had no significant association with tumor stage, Gleason score, and PSA levels [27]. The third study $(n=193)$ found that higher mtDNAcn in peripheral blood leukocytes was associated with more advanced tumor stage, higher Gleason score, and higher PSA levels
[28]. The reasons for the inconsistency include random errors due to the small sample sizes of these prior studies, variations in the protocols for assigning Gleason score and tumor stage, variations in the measurements of PSA levels and mtDNAcn, and diverse genetic background of the study populations (e.g., the second study used a mixture of Caucasians and African Americans and the third study used a Chinese population from two hospitals).

Our study provided the first evidence that low mtDNAcn in peripheral blood leukocytes might be associated with an increased risk of disease progression of localized PCa, although after adjustment of age, BMI, smoking status, pack-year, D'Amico risk groups, and treatments, the associations were borderline significant, likely due to the relatively small number of events among localized PCa. Consistent with our study, a previous report among breast cancer patients found that reduced mtDNAcn in tumor tissues was associated with increased 
risks of disease progression and all-cause mortality [32]. On the other hand, Grupp et al. [33] analyzed the expression of MTC02, a $60 \mathrm{kDa}$ non-glycosylated protein component of mitochondria, in the tumor tissues of PCa patients via tissue microarray, and found that high mitochondria content, as indicated by increased expression of MTC02, was associated with an elevated risk of biochemical recurrence. It should be pointed out that our study and Grupp's study used different measures (i.e., mtDNAcn vs. MTC02 expression) and therefore this discrepant result should be interpreted with caution.

Our findings are biologically plausible for several reasons. First, a decrease in mtDNAcn leads to insufficient oxidative phosphorylation and greater generation of adenosine triphosphate (ATP) by glycolysis [34]. These changes could result in a stronger tolerance to hypoxia and reduced dependence on mitochondrial oxidative phosphorylation, thus conferring growth advantage to tumor cells $[26,31,35]$. Second, a decrease in mtDNAcn may promote cells to become resistant to apoptosis $[36,37]$ through the activation of phosphatidylinositol 3-kinase (PI3K)/Akt2 signaling [35, 38, 39], and resistance to apoptosis contributes to cancer progression [40-42]. Third, mtDNA depletion in PCa cells induces epithelialmesenchymal transition $[43,44]$, which plays an important role in tumor progression [45]. Finally, one study using PCa cell lines showed that reduction in mtDNAcn shifted androgen-dependent $\mathrm{PCa}$ cells to a more aggressive androgen-independent phenotype [24].

The findings from our study may have important clinical implications for the management of localized PCa patients. Our results suggested that pre-treatment mtDNAcn in peripheral blood leukocytes could serve as a marker to distinguish between indolent and aggressive PCa. Additionally, our results indicated that low mtDNAcn predicted the presence of aggressive tumors among patients with a negative PSA test (i.e., $\leq 4 \mathrm{ng} / \mathrm{ml}$ ). Further, it might predict disease progression independent of commonly used clinical parameters including Gleason score, clinical stage, and PSA. Therefore, mtDNAcn in peripheral blood leukocytes might be included as an independent factor in prediction models to better distinguish between indolent and aggressive $\mathrm{PCa}$ and to predict prognosis of $\mathrm{PCa}$ patients in order to minimize over-treatment of indolent $\mathrm{PCa}$ and under-treatment of aggressive PCa.

Our study has several strengths. It is the largest study to date (our sample size is over 7 times larger than the combined sample size of the three previous studies) to investigate whether mtDNAcn in peripheral blood leukocytes was associated with aggressive features of localized PCa, and the first study to investigate its association with disease progression. We were able to show the associations after adjustment of comprehensive potential confounding factors including age, BMI, smoking, pack-year, clinical and histopathological characteristics of the tumor, and treatments. Consistent and standard protocols were used in the measurements of mtDNAcn, histopathological diagnoses, treatment procedures, and collection of covariates for all of our study patients. Despite these strengths, our study was limited to non-Hispanic white men, and caution should be taken when generalizing the results to other ethnic groups.

In conclusion, the results from this large study of localized PCa patients suggested that low mtDNAcn in peripheral blood leukocytes at diagnosis was associated with aggressive $\mathrm{PCa}$ and could further predict disease progression beyond commonly used clinical parameters. Future studies are needed to validate our results in more diverse $\mathrm{PCa}$ patient populations.

\section{MATERIALS AND METHODS}

\section{Study population and data collection}

The recruitment and data collection for our study population have been previously reported [46]. Briefly, our study recruited 1,859 non-Hispanic white men with previously untreated localized PCa who were treated at The University of Texas MD Anderson Cancer Center. Among them, measurements of mtDNAcn in peripheral blood leukocytes in blood samples collected at diagnosis were available for 1,751 (94\%) patients. At registration, patients filled in questionnaires on demographics, BMI, and smoking history. Study staff conducted detailed chart review of medical records to abstract clinical data including diagnosis date, PSA level at diagnosis, biopsy-proven Gleason score, clinical tumor stage, and treatments. Follow-up information on disease progression was abstracted for patients who received active treatments $(n=1,399)$, and this information was available for 1,266 $(90.5 \%)$ patients. Follow-up PSA tests were conducted every 3 months for patients with locally advanced tumor (T3a, T3b, and T4) and every 3-6 months for 5 years, then every 6-12 months for 5 years, then annually for patient post definitive therapy. This study was approved by the MD Anderson Cancer Center Institutional Review Board, and written informed consent was obtained from each participant.

\section{Definition of aggressiveness and disease progression}

We defined aggressiveness according to the D'Amico risk criteria [23]: low-risk form of PCa (Gleason score $\leq 6$ and clinical stage T1-T2 $a$ and PSA $\leq 10 \mathrm{ng} / \mathrm{ml}$ ), intermediate-risk form (Gleason score of 7 and/or stage T2b and/or PSA level $>10$ and $\leq 20 \mathrm{ng} / \mathrm{mL}$ ), and highrisk form (Gleason score $\geq 8$ or clinical stage T2c-T4 or PSA $>20 \mathrm{ng} / \mathrm{ml}$ ). Disease progression was defined as biochemical recurrence (BCR), or presence of local recurrence or distant metastasis, whichever came first, because some cases presented with local recurrence or distant metastasis but no records of a PSA increase. 
A BCR was defined as a PSA $\geq 0.2 \mathrm{ng} / \mathrm{mL}$ with a second confirmatory level of PSA $>0.2 \mathrm{ng} / \mathrm{mL}$ for patients treated with radical prostatectomy, as recommended by the American Urological Association Prostate Cancer Guidelines Panel [47], or a PSA rise of $2 \mathrm{ng} / \mathrm{mL}$ or more above the nadir PSA for patients who received radiotherapy, according to the Radiation Therapy Oncology Group-American Society for Therapeutic Radiology and Oncology (RTOG-ASTRO) consensus [48].

\section{Measurements of mtDNA copy number}

The relative mtDNAcn in peripheral blood leukocytes was measured by a quantitative reverse transcriptionPCR-based method as previously described [49, 50]. In brief, two pairs of primers were used in the two steps of relative quantification of mtDNAcn. One primer pair (ND1-F, 5'-CCCTAAAACCCGCCACATCT-3'; ND1-R, 5'-GAGCGATGGTGAGAGCTAAGGT-3') was used to amplify the mitochondrial ND1 gene, and the other primer pair (HGB-1, 5'-GTGCACCTGACTCCTGAGGAGA-3'; HGB-2, 5'-CCTTGATACCAACCTGCCCAG-3') was used to amplify the single-copy nuclear $H G B$ gene.

In the first step, the ratio of the copy number of mitochondrial $N D 1$ gene to the nuclear $H G B$ gene was determined for each sample from standard curves. This ratio is proportional to the mtDNAcn in each cell. The ratio for each sample was then normalized to a calibrator DNA in order to standardize between different runs. The calibrator DNA is from a genomic DNA sample of a healthy control. The PCR mixture of $14 \mu \mathrm{l}$ contained $1 \times$ SYBR Green PCR Master Mix (Applied Biosystems, Foster City, CA), 215 nM ND1-F and ND1-R (or HGB-1 and HGB-2) primers, and $4 \mathrm{ng}$ of genomic DNA. The thermal cycling conditions for the mitochondrial ND1 gene amplification were $95^{\circ} \mathrm{C}$ for $10 \mathrm{~min}$, followed by 40 cycles of $95^{\circ} \mathrm{C}$ for $15 \mathrm{~s}$ and $60^{\circ} \mathrm{C}$ for $1 \mathrm{~min}$; for the nuclear $H G B$ gene amplification, the cycling conditions were $95^{\circ} \mathrm{C}$ for $10 \mathrm{~min}$, followed by 40 cycles of $95^{\circ} \mathrm{C}$ for $15 \mathrm{~s}$ and $56^{\circ} \mathrm{C}$ for $1 \mathrm{~min}$. All samples were plated in duplicates on 384-well plates and run on an Applied Biosystems 7900HT Sequence Detection System. The PCRs for ND1 and $H G B$ genes were performed on separate 384-well plates with the same samples in the same well positions to avoid possible position effects.

A standard curve of a serially diluted reference DNA, one negative control and one calibrator DNA were included in each run. For each standard curve, one reference DNA sample was serially diluted 1:2 to produce a seven-point standard curve between 0.3125 and $20 \mathrm{ng}$ of DNA. The $R^{2}$ for each standard curve was 0.99 or greater. Standard deviations for the cycle of threshold value were acceptable at 0.25 . Otherwise, the test was repeated. The intra-assay coefficient of variation for all samples varies from 0.1 to $35 \%$ with an overall mean of $5 \%$.

\section{Statistical analysis}

Patients with different host and clinical characteristics at baseline were compared by levels of mtDNAcn using analysis of variance (ANOVA). mtDNAcn was categorized into three groups according to the tertiles in the study population. For the associations between mtDNAcn and aggressiveness of $\mathrm{PCa}$, we calculated odds ratios (ORs) with 95 percent confidence intervals (95\% CIs) using multinomial logistic regression with the low-risk $\mathrm{PCa}$ as the reference outcome after adjustment of age $(<55,55-65$, and $>65$ yrs. $)$, BMI (normal/underweight, overweight, and obese), smoking status (never, former, and current smokers), and pack-year (continuous) based on a priori knowledge. Patients with missing covariates were not included in the multivariate analyses. In addition, we used restricted cubic spline to model the potential non-linear relationship between mtDNAcn and aggressiveness of $\mathrm{PCa}$; four knots were used and the $99^{\text {th }}$ percentile $(2.195)$ was chose as the reference level.

Time to event for the analysis of disease progression was calculated from the date of diagnosis to the date of disease progression, death, or the latest follow-up, whichever came first. We used Kaplan-Meier survival analysis and log-rank tests to assess the differences in disease progression across different levels of mtDNAcn. We used Cox proportional hazards model to estimate hazard ratios (HRs) and 95\% CIs after adjustment of age, BMI, smoking status, pack-year, D' Amico risk groups (low-risk group, intermediate-risk group, and high-risk group), and primary treatment (radical prostatectomy, radiotherapy, active surveillance or unknown, and other treatments). All statistical analyses were performed using STATA version 10 (StataCorp, College Station, TX, USA). A $P$ value $<0.05$ (two-sided) was considered statistically significant.

\section{CONFLICTS OF INTEREST}

The authors declare no conflicts of interest.

\section{GRANT SUPPORT}

This study was financially supported by the NCI (grant CA140388), the Cancer Prevention and Research Institute of Texas (CPRIT, grant RP140556), and the MD Anderson Cancer Center institutional support for the Center for Translational and Public Health Genomics.

\section{REFERENCES}

1. Nunnari J, Suomalainen A. Mitochondria: in sickness and in health. Cell. 2012; 148:1145-1159. 
2. Weinberg SE, Chandel NS. Targeting mitochondria metabolism for cancer therapy. Nat Chem Biol. 2015; 11:9-15.

3. Chan DC. Mitochondria: dynamic organelles in disease, aging, and development. Cell. 2006; 125:1241-1252.

4. Satoh M, Kuroiwa T. Organization of multiple nucleoids and DNA molecules in mitochondria of a human cell. Exp Cell Res. 1991; 196:137-140.

5. Veltri KL, Espiritu M, Singh G. Distinct genomic copy number in mitochondria of different mammalian organs. J Cell Physiol. 1990; 143:160-164.

6. Clay Montier LL, Deng JJ, Bai Y. Number matters: control of mammalian mitochondrial DNA copy number. J Genet Genomics. 2009; 36:125-131.

7. Penta JS, Johnson FM, Wachsman JT, Copeland WC. Mitochondrial DNA in human malignancy. Mutat Res. 2001; 488:119-133.

8. Wallace DC. Mitochondrial DNA sequence variation in human evolution and disease. Proc Natl Acad Sci U S A. 1994; 91:8739-8746.

9. Van Houten B, Woshner V, Santos JH. Role of mitochondrial DNA in toxic responses to oxidative stress. DNA Repair (Amst). 2006; 5:145-152.

10. Lee HC, Wei YH. Mitochondrial biogenesis and mitochondrial DNA maintenance of mammalian cells under oxidative stress. Int J Biochem Cell Biol. 2005; 37:822-834.

11. Higuchi M. Roles of Mitochondrial DNA Changes on Cancer Initiation and Progression. Cell Biol (Henderson, NV). 2012; 1.

12. Chatterjee A, Mambo E, Sidransky D. Mitochondrial DNA mutations in human cancer. Oncogene. 2006; 25:4663-4674.

13. El-Hattab AW, Scaglia F. Mitochondrial DNA depletion syndromes: review and updates of genetic basis, manifestations, and therapeutic options. Neurotherapeutics. 2013; 10:186-198.

14. Lee HC, Yin PH, Lin JC, Wu CC, Chen CY, Wu CW, Chi CW, Tam TN, Wei YH. Mitochondrial genome instability and mtDNA depletion in human cancers. Ann N Y Acad Sci. 2005; 1042:109-122.

15. Jessie BC, Sun CQ, Irons HR, Marshall FF, Wallace DC, Petros JA. Accumulation of mitochondrial DNA deletions in the malignant prostate of patients of different ages. Exp Gerontol. 2001; 37:169-174.

16. Arnold RS, Sun CQ, Richards JC, Grigoriev G, Coleman IM, Nelson PS, Hsieh CL, Lee JK, Xu Z, Rogatko A, Osunkoya AO, Zayzafoon M, Chung L, et al. Mitochondrial DNA mutation stimulates prostate cancer growth in bone stromal environment. Prostate. 2009; 69:1-11.

17. Jerónimo $\mathrm{C}$, Nomoto $\mathrm{S}$, Caballero $\mathrm{OL}$, Usadel $\mathrm{H}$, Henrique R, Varzim G, Oliveira J, Lopes C, Fliss MS, Sidransky D. Mitochondrial mutations in early stage prostate cancer and bodily fluids. Oncogene. 2001; 20:5195-5198.

18. Petros JA, Baumann AK, Ruiz-Pesini E, Amin MB, Sun CQ, Hall J, Lim S, Issa MM, Flanders WD, Hosseini SH, Marshall FF, Wallace DC. mtDNA mutations increase tumorigenicity in prostate cancer. Proc Natl Acad Sci U S A. 2005; 102:719-724.

19. American Cancer Society . Cancer Facts \& Figures 2015. Atlanta: American Cancer Society. 2015.

20. Howlader N NA, Krapcho M, Garshell J, Miller D, Altekruse SF, Kosary CL, Yu M, Ruhl J, Tatalovich Z, Mariotto A, Lewis DR, Chen HS, Feuer EJ, Cronin KA. SEER Cancer Statistics Review 1975-2011 (National Cancer Institute Bethesda, MD based on November 2013 SEER data submission, posted to the. SEER web site. April 2014; http://seer.cancer.gov/csr/1975_2011/.

21. Welch HG, Albertsen PC. Prostate cancer diagnosis and treatment after the introduction of prostate-specific antigen screening: 1986-2005. J Natl Cancer Inst. 2009; 101:1325-1329.

22. Rodrigues G, Warde P, Pickles T, Crook J, Brundage M, Souhami L, Lukka H. Pre-treatment risk stratification of prostate cancer patients: A critical review. Can Urol Assoc J. 2012; 6:121-127.

23. D'Amico AV, Whittington R, Malkowicz SB, Schultz D, Blank K, Broderick GA, Tomaszewski JE, Renshaw AA, Kaplan I, Beard CJ, Wein A. Biochemical outcome after radical prostatectomy, external beam radiation therapy, or interstitial radiation therapy for clinically localized prostate cancer. Jama. 1998; 280:969-974.

24. Higuchi M, Kudo T, Suzuki S, Evans TT, Sasaki R, Wada Y, Shirakawa T, Sawyer JR, Gotoh A. Mitochondrial DNA determines androgen dependence in prostate cancer cell lines. Oncogene. 2006; 25:1437-1445.

25. Moro L, Arbini AA, Marra E, Greco M. Mitochondrial DNA depletion reduces PARP-1 levels and promotes progression of the neoplastic phenotype in prostate carcinoma. Cell Oncol. 2008; 30:307-322.

26. Cook CC, Kim A, Terao S, Gotoh A, Higuchi M. Consumption of oxygen: a mitochondrial-generated progression signal of advanced cancer. Cell Death Dis. 2012; 3:e258.

27. Koochekpour S, Marlowe T, Singh KK, Attwood K, Chandra D. Reduced mitochondrial DNA content associates with poor prognosis of prostate cancer in African American men. PLoS One. 2013; 8:e74688.

28. Zhou W, Zhu M, Gui M, Huang L, Long Z, Wang L, Chen H, Yin Y, Jiang X, Dai Y, Tang Y, He L, Zhong K. Peripheral blood mitochondrial DNA copy number is associated with prostate cancer risk and tumor burden. PLoS One. 2014; 9:e109470.

29. Wu CW, Yin PH, Hung WY, Li AF, Li SH, Chi CW, Wei YH, Lee HC. Mitochondrial DNA mutations and 
mitochondrial DNA depletion in gastric cancer. Genes Chromosomes Cancer. 2005; 44:19-28.

30. Wang Y, Liu VW, Xue WC, Cheung AN, Ngan HY. Association of decreased mitochondrial DNA content with ovarian cancer progression. Br J Cancer. 2006; 95:1087-1091.

31. Simonnet H, Alazard N, Pfeiffer K, Gallou C, Beroud C, Demont J, Bouvier R, Schagger H, Godinot C. Low mitochondrial respiratory chain content correlates with tumor aggressiveness in renal cell carcinoma. Carcinogenesis. 2002; 23:759-768.

32. Yu M, Zhou Y, Shi Y, Ning L, Yang Y, Wei X, Zhang N, Hao X, Niu R. Reduced mitochondrial DNA copy number is correlated with tumor progression and prognosis in Chinese breast cancer patients. IUBMB Life. 2007; 59:450-457.

33. Grupp K, Jedrzejewska K, Tsourlakis MC, Koop C, Wilczak W, Adam M, Quaas A, Sauter G, Simon R, Izbicki JR, Graefen M, Huland H, Schlomm T, et al. High mitochondria content is associated with prostate cancer disease progression. Mol Cancer. 2013; 12:145.

34. Shadel GS. Expression and maintenance of mitochondrial DNA: new insights into human disease pathology. Am J Pathol. 2008; 172:1445-1456.

35. Pelicano H, Xu RH, Du M, Feng L, Sasaki R, Carew JS, Hu Y, Ramdas L, Hu L, Keating MJ, Zhang W, Plunkett W, Huang P. Mitochondrial respiration defects in cancer cells cause activation of Akt survival pathway through a redoxmediated mechanism. J Cell Biol. 2006; 175:913-923.

36. Chandel NS, Schumacker PT. Cells depleted of mitochondrial DNA (rho0) yield insight into physiological mechanisms. FEBS Lett. 1999; 454:173-176.

37. Higuchi M, Aggarwal BB, Yeh ET. Activation of CPP32like protease in tumor necrosis factor-induced apoptosis is dependent on mitochondrial function. J Clin Invest. 1997; 99:1751-1758.

38. Suzuki S, Naito A, Asano T, Evans TT, Reddy SA, Higuchi M. Constitutive activation of AKT pathway inhibits TNF-induced apoptosis in mitochondrial DNA-deficient human myelogenous leukemia ML-1a. Cancer Lett. 2008; 268:31-37.

39. Moro L, Arbini AA, Yao JL, di Sant'Agnese PA, Marra E, Greco M. Mitochondrial DNA depletion in prostate epithelial cells promotes anoikis resistance and invasion through activation of PI3K/Akt2. Cell Death Differ. 2009; 16:571-583.

40. Kerr JF, Wyllie AH, Currie AR. Apoptosis: a basic biological phenomenon with wide-ranging implications in tissue kinetics. Br J Cancer. 1972; 26:239-257.
41. Wong RS. Apoptosis in cancer: from pathogenesis to treatment. J Exp Clin Cancer Res. 2011; 30:87.

42. Lowe SW, Lin AW. Apoptosis in cancer. Carcinogenesis. 2000; 21:485-495.

43. Naito A, Cook CC, Mizumachi T, Wang M, Xie CH, Evans TT, Kelly T, Higuchi M. Progressive tumor features accompany epithelial-mesenchymal transition induced in mitochondrial DNA-depleted cells. Cancer Sci. 2008; 99:1584-1588.

44. Guha M, Srinivasan S, Ruthel G, Kashina AK, Carstens RP, Mendoza A, Khanna C, Van Winkle T, Avadhani NG. Mitochondrial retrograde signaling induces epithelialmesenchymal transition and generates breast cancer stem cells. Oncogene. 2014; 33:5238-5250.

45. Huber MA, Kraut N, Beug H. Molecular requirements for epithelial-mesenchymal transition during tumor progression. Curr Opin Cell Biol. 2005; 17:548-558.

46. He Y, Gu J, Strom S, Logothetis CJ, Kim J, Wu X. The prostate cancer susceptibility variant rs2735839 near KLK3 gene is associated with aggressive prostate cancer and can stratify gleason score 7 patients. Clin Cancer Res. 2014; 20:5133-5139.

47. Cookson MS, Aus G, Burnett AL, Canby-Hagino ED, D'Amico AV, Dmochowski RR, Eton DT, Forman JD, Goldenberg SL, Hernandez J, Higano CS, Kraus SR, Moul JW, et al. Variation in the definition of biochemical recurrence in patients treated for localized prostate cancer: the American Urological Association Prostate Guidelines for Localized Prostate Cancer Update Panel report and recommendations for a standard in the reporting of surgical outcomes. J Urol. 2007; 177:540-545.

48. Roach M, 3rd, Hanks G, Thames H, Jr, Schellhammer P, Shipley WU, Sokol GH, Sandler H. Defining biochemical failure following radiotherapy with or without hormonal therapy in men with clinically localized prostate cancer: recommendations of the RTOG-ASTRO Phoenix Consensus Conference. Int J Radiat Oncol Biol Phys. 2006; 65:965-974.

49. Xing J, Chen M, Wood CG, Lin J, Spitz MR, Ma J, Amos CI, Shields PG, Benowitz NL, Gu J, de Andrade M, Swan GE, Wu X. Mitochondrial DNA content: its genetic heritability and association with renal cell carcinoma. J Natl Cancer Inst. 2008; 100:1104-1112.

50. Kaaman M, Sparks LM, van Harmelen V, Smith SR, Sjolin E, Dahlman I, Arner P. Strong association between mitochondrial DNA copy number and lipogenesis in human white adipose tissue. Diabetologia. 2007; 50:2526-2533. 